\title{
Comparison of Concepts within the Residential Colleges in Taiwan
}

\author{
Yueh-Luen Hu, Gregory S. Ching, and Chao-Hsiang Hung
}

\begin{abstract}
Within the model of residential colleges, it is said that a fruitful educational experience is not limited to rigorous academics, but must also include healthy non-academic activities that allows the development of the sense of community and civic responsibility within the students. In Taiwan, after around five years of implementation of the residential college model of education, the current paper shall attempt to discuss and analyze the inherent concepts within these colleges. Using the qualitative approach of structured conceptualization method or concept mapping; 40 participants comprising of faculty, administrators, and students from the 4 key residential colleges in Taiwan are interviewed and surveyed. After 4 separate focus group discussions, a set of indicators is formulated as the students' abilities; that successfully describe the concepts within the residential colleges. In addition, to make the analysis simpler, the concept mapping procedures also resulted in the presentation of a set of graphical representation of the indicators. Further implications are also provided upon comparison of the perceived importance and performance of the students. In sum, residential colleges promise a shift in the way on how students in Taiwan are developed and shaped. However, more empirical and comparative studies should be undertaken to further the understanding of the actual effects and impacts of such program on the overall students' educational outcome.
\end{abstract}

Index Terms-Residential colleges, students' abilities, concept mapping, core competencies, liberal arts education

\section{INTRODUCTION}

The collegial models of academic system vary differently between the East and the West. In China, traditional academic systems are called Chinese Academies; wherein this is more commonly known as the concept of Residential Colleges within the Western culture. Although the names are different, however, both of these academic systems practice the concept of residential learning community. Within the west, residential colleges can be traced back from the early ages of prototype colleges of the University of Oxford and Cambridge of the United Kingdom. During the middle ages, students' enrolment and management system are quite different from what universities that we are experiencing today. In those days, the colleges are not only a place for teaching and learning, but it is a place where the teachers and students lived. In essence, the classical residential college is

Manuscript received May 10, 2014; revised August 11, 2014. This work was supported in part by the Taiwan Ministry of Science and Technology under Grant NSC 101-2410-H-004-168-MY2.

Yueh-Luen $\mathrm{Hu}$ and Chao-Hsiang Hung are with the Department of Education, National ChengChi University, Taiwan (e-mail: joyhu@nccu.edu.tw,aka0518@gmail.com).

Gregory S. Ching is with the Graduate School of Educational Leadership and Development, Fu Jen Catholic University, Taiwan (e-mail: gregory_ching@yahoo.com). characterized by one factor, which is the notion that faculty residing amongst their students [1]. Therefore, we can establish that the concept of residential colleges, basically builds on the notion that freshmen entering their university years should feel welcomed by senior students and faculty. As the years progress, their relationships and activities should help foster and develop a sense of belongingness to the institution; in a way, a sense of ownership of its (the institution's) collective values. This is not only important for creating a rich and multi-faceted educational experience, but it is also critical in building a form of counselling system to students who are lagging behind not only in academic, but also in their social and/or personal development [2].

To understand the inherent concepts behind the establishment (and revival) of residential colleges in Taiwan, the current presentation shall use the structured conceptualization method or more commonly known as concept mapping to depict a graphical representation if the concepts. As of the moment, four key universities (National Tsing Hua University, National Chung Cheng University, National Cheng-chi University, and Tunghai University) in Taiwan are utilizing this concept of residential colleges. In their collective ideals and practices, all of these colleges in one way or another noted that a quality education experience is not limited to rigorous academics, but should also integrate healthy non-academic activities that instil a sense of community and civic responsibility amongst students.

\section{Methodology}

The current study utilizes the qualitative process of structured conceptualization method of concept mapping in order to capture the collective thinking within the establishment of these residential colleges in Taiwan. Structured conceptualization method is considered to be both a descriptive and interpretive method used for making sense of results [3].

To define, concept mapping is a method of structured conceptualization specifically designed for used by groups of individuals (with a common goal) to develop a conceptual framework [4]-[6]. More importantly, concept mapping is use in conceptualizing (organizing in concepts) open-ended responses within focus group discussions [7], wherein these opinions from various stakeholders are being considered and generalized [8], [9]. In sum, structured conceptualization or concept mapping can be described as a sequence of concrete operationally-defined steps and which yields a conceptual (graphical) representation [10].

Concept mapping usually follows six procedures: 1) Preparation includes the selection of participants and development of the theme for the conceptualization; 2) 
Generation (items generation); 3) Structuring (or sorting); 4) Representation (graphical); 5) Interpretation; and 6) Utilization. The following section shall discuss how the procedure of concept mapping evolves within the study.

\section{A. Preparation Stage}

In the preparation stage, during this procedure participants are first selected. This is a very important stage; most concept mapping process includes participants comprising of stakeholders (immediate concerned individuals) within the residential colleges. More importantly, it is assumed that there is an identifiable group responsible for guiding the discussions. Hence, assigning the appropriate participants is crucial to the success and accuracy of the entire process [11].

With the current study, in order to gather data from all of the stakeholders of the residential college, the researchers decided that the participants of the concept mapping process to be the residential college faculty, administrators, and students. Within the study, a total of fortyparticipants from four key residential colleges comprising of seven administrators (including dormitory counselors, class advisers, and the like), eight teachers, and twenty-five students participated in the entire concept mapping process.

Table I shows the breakdown of the participants by school and positions. Please note that the administrators and faculty participants are carefully selected so as to gather a more comprehensive and holistic view of the residential colleges. Besides the selection of participants, the core focus (or theme) of the concept mapping process is also to develop key concepts through the discussions with the administrators and faculties. Hence, key core objectives (discussion points) are established, namely: a) what are competencies being developed within the residential colleges? b) Describe the typical (or ideal) abilities of a residential college graduate.

TABLE I: PARTICIPANTS' DEMOGRAPHY $(N=40)$

\begin{tabular}{lccc}
\hline \hline \multicolumn{1}{c}{ Institution } & Administrators & Faculty & Students \\
\hline National Tsing Hua University (NTHU) & 3 & 2 & 5 \\
National Chung Cheng University (CCU) & 1 & 3 & 8 \\
National Cheng-chi University (NCCU) & 2 & 2 & 6 \\
Tunghai University (THU) & 1 & 1 & 6 \\
\hline \hline
\end{tabular}

\section{B. Generation Stage}

Within the statement generation stage (somewhat very similar to a brainstorming session), participants are contacted and informed of the date and venue of the session. Within each of the session, participants are asked to generate ideas with relation to the topic of interest. More specifically, what are the competencies being developed within the residential colleges? Similarly, participants are asked to describe the typical (or ideal) abilities of a residential college graduate.

In this stage, participants are encouraged to generate lots of statements and are told that there should be no criticism or discussion regarding the legitimacy of the statements which are generated during the session.

Participants are also encouraged to ask for clarification of any unfamiliar terms so as to ensure that all participants are on the same level of understanding. In the end, a total of 4 statement generation (focus group) sessions are accomplished; one for each of the residential college. Once the final set of statements has been generated and collated from all the sessions, the researchers re-examine the statements for consistency of wordings. More importantly, the statements are check redundancy and centrality of focus. Afterwards, these statements are then sent back to the administrators and faculty for a final content validity check.

\section{Structuring Stage}

Within the concept mapping process, analysis is accomplished by means of organization and representation activities; wherein the core data for a concept map come from the unstructured or free sort or also known as the structuring of the statement procedures [12], [13]. During this stage, each of the participants is given the task to assigned and group the generated statements into piles, which the participants think is meaningful (see Fig. 1 for a graphical representation of the resulting outcome). Participants are free to use as few or as many piles as they think necessary. Participants are also reminded that placing all of the statements into one pile is not allowed.

After the piles are finalized, participants are asked to provide a name and their corresponding description to each of the pile. In addition, a statement rating survey is also provided to the participants. Here the participants are requested to rate each of the statements through a Likert type scale of 1 to 10 ( 1 as the least and 10 as the most) for their perceived importance and performance of the students.

\section{Hypothetical Sort}

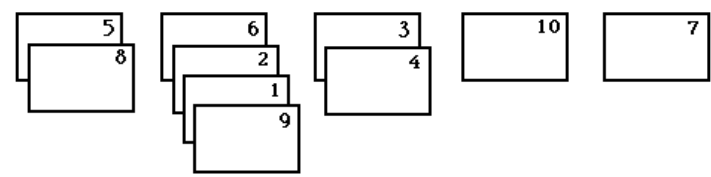

Fig. 1. Hypothetical sort (Source: http://www.socialresearchmethods.net).

\section{Representation Stage}

Representation of the statement stage can also be thought as the data analysis stage. In this stage, data in the sorted piles are converted to a 0 and 1 co-occurrence matrix [13], which has as many rows and columns as the statements are. 1 is entered into a cell if the row and column statement pair is found in the same pile and a 0 is entered if the statement is not.

These matrices are then summed across all participants, yielding a similarity matrix that indicates the number of participants that places each pair of statements together. This is then computed through the use of a summed square similarity matrix using multidimensional scaling (MDS) analysis [14], [15], which takes dissimilarity data and represents them as distances in Euclidean space.

\section{E. Interpretation and Utilization Stage}

After the MDS solutions are generated, additional information from clusters and ratings are formulated. For the clusters, each of the statements would generate a unique $\mathrm{X}$ and $\mathrm{Y}$ value. When plotted in a bivariate plot, these points constitute the basic point map form of the concept map. The MDS X, Y values are actually the input for hierarchical cluster analysis using Ward's algorithm [16], [17], which has the effect of partitioning the MDS statement map 
hierarchically into non-overlapping clusters.

Lastly, these clusters are then superimposed with each other forming meaningful patterns, thus, depicting the different conceptual factors inherent within the residential colleges. These stages are computed through the use of the Concept System; a computer program which facilitates the entry and analysis of concept mapping data developed by Trochim [18]. The program is capable of using a combination of multidimensional scaling and cluster analysis techniques to represent graphically the conceptual relationships underlying the data, which is considered quite useful.

\section{RESULTS AND DISCUSSIONS}

During the generation stage, a group of indicators is formulated, namely the abilities; that would best describe the inherent concepts within the residential colleges. The abilities are composed of twenty-seven statements describing key competencies a residential college graduate should possess. The abilities also formed the six proposed factors, which are as follows: Team work, Independent, Multidisciplinary skills, Civic-mindedness, Open-mindedness, and Professional skills. Fig. 2 shows the interpretation stage results, wherein individual statements (represented as points) on the map form into the 6 factors (or clusters), which are grouped using the Ward's hierarchical cluster analysis [16], [17] on the XY coordinate provided by the MDS.

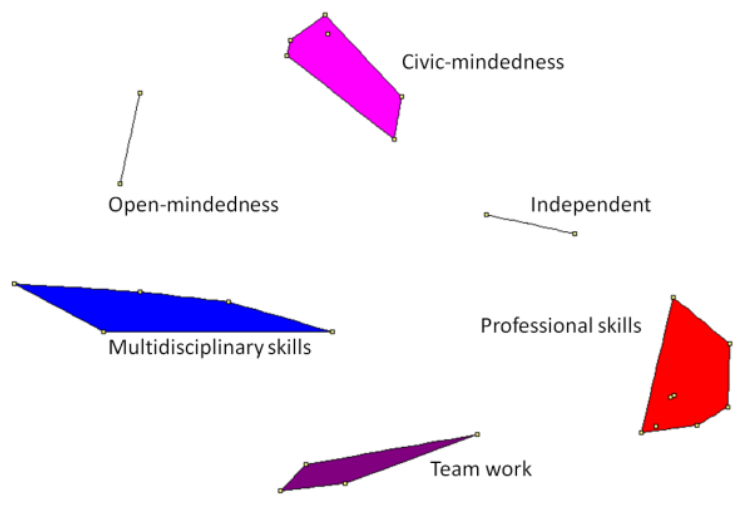

Fig. 2. Point-cluster map.

To better understand these factors, the following section shall provide the various operating definitions gathered from the results of the concept mapping development process.

Civic-mindedness: Within the residential college model of education, students are taught to have a better understanding and respect of the law. Moreover, today's students are tomorrow's leader, hence, various civic duties such as upholding social justice and participation in public affairs are encourage. In addition, students are also trained to have a global perspective and show concerns for international affairs. More importantly, promote activities towards a sustainable environment. Cluster statements include 6 items, namely: Actively participate in public affairs; Vote according to own opinion; Have global perspective and show concerns for international affairs; Have the willingness and the ability to uphold social justice; Participate and promote activities towards sustainable environment; and Have a basic knowledge of the law and abides by it.
Open-mindedness: Besides having the sense for civic engagement, students should also be able to remember their roots. Hence, residential colleges promote the appreciation of one's own culture and identity; while at the same time have the equal respect for others who are different from oneself. Cluster statements include 2 items, namely: Respect and appreciate a culture different from own and Recognizes one's own culture and identity.

Independent: Another key feature of the residential college is to encourage the student to think rationally and independently, while at the same time think responsively and objectively. Expression of one's opinion is encouraged; however, students should also remember the responsibility that comes with it. Cluster statements include 2 items, namely: Ability to think rationally in issues wherein the society has a difference of opinion and Able to use effective critical thinking skills.

Multidisciplinary skills: The residential colleges also encouraged students to become flexible. Being able to appreciate not only the complexity of science, but also the beauty and values of arts can offer. Cluster statements include 3 items, namely: Able to explore multidisciplinary knowledge; Set goals and learn actively; Profound and comprehensive self-awareness; Adapts \& lives well in a new environment; and Appreciates \& values any work of art.

Team work: As in today's environment, no one person can survive alone. Hence, students are encouraged to become both an outstanding leader and effective member. Cluster statements include 4 items, namely: Able to work and interact well with group members; Have effective leadership skills; Possess good inter-personal skills; and Possess good communication skills.

Professional skills: Residential colleges also encourage students to excel on competencies necessary for future employment. As Taiwan performs globally having an effective English language competency is also encouraged. Students should be competent in information technology skills. In addition, students are also required to attain a functional level in the four basic skills of listening, speaking, reading, and writing in a foreign language; English. Cluster statements include 8 items, namely: Is employable and have competitiveness; Able to use the scientific method to solve problems; Have proficient writing skills; Have English reading skills; Have professional skills; Possess good English listening and speaking skills; Knows how to work around with a computer; and Have mathematical/computational skills.

To determine how important the factors are and how do students perform within these factors; a 10 point Likert type ( 1 as the least and 10 as the most) survey was also accomplished by the participants. Here participants are tasked to rank the perceived importance and manifestation (or performance) of the students within each statements. Table II shows the results of the survey. Results show that the factor team work ranks highest both in importance $(M=8.00)$ and actual students' performance $(M=7.73)$. This is followed by Independent $(M=7.64)$, Multidisciplinary skills $(M=7.58)$, Civic-mindedness $(M=7.35)$, Open-mindedness $(M=7.30)$, and Professional skills $(M=5.51)$ as the least. Note that the importance decrease almost 2 points in the Professional skills 
upon comparing with the other factors.

TABLE II: IMPORTANCE AND PERFORMANCE OF THE FACTORS $(N=40)$

\begin{tabular}{lcr|cccc}
\hline \hline Clusters & Rank & Impt. & Rank & Perf. & Mean Difference (M-I) \\
\hline Team work & 1 & 8.00 & 1 & 7.73 & -0.27 \\
Independent & 2 & 7.64 & 3 & 7.16 & -0.48 \\
Multidisciplinary skills & 3 & 7.58 & 4 & 6.90 & -0.68 \\
Civic-mindedness & 4 & 7.35 & 5 & 6.60 & -0.75 \\
Open-mindedness & 5 & 7.30 & 2 & 7.18 & -0.13 \\
Professional skills & 6 & 5.51 & 6 & 5.91 & 0.40 \\
\hline \hline
\end{tabular}

As for the students' actual performance, the participants also ranks the highest is Team work followed by Open-mindedness $\quad(M=7.18), \quad$ Independent $\quad(M=7.16)$, Multidisciplinary skills $(M=6.90), \quad$ Civic-mindedness $(M=6.60)$, and Professional skills $(M=5.91)$ as the least. It is noted that both importance and performance ratings ranked Professional skills as the least, since residential colleges are more focused on factors that are beyond the students' Professional skills. Furthermore, Table II also shows the mean differences between importance and performance. Results show that participants rank the importance as much higher than the actual performance of the students (except in the factor Professional skills), suggesting that there are still room for improvement.

In addition, in order to have a more visual representation of the importance and performance of the factors; cluster rating maps are also created. Cluster rating map is created by means of overlaying the average ratings of importance (and later performance) on the cluster map (please see Fig. 3 and Fig. 4). Note that the layers represent the importance of the cluster. Hence, the more layers the cluster has (or the higher the layers are), means the greater the importance of the cluster. Similarly, the higher the makers at the points, the higher the importance (and later performance in Fig. 4) are.

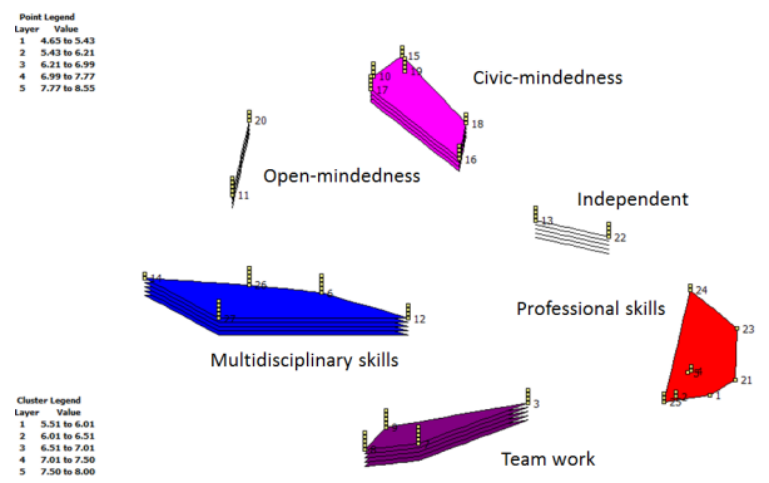

Fig. 3. Cluster rating map (importance).

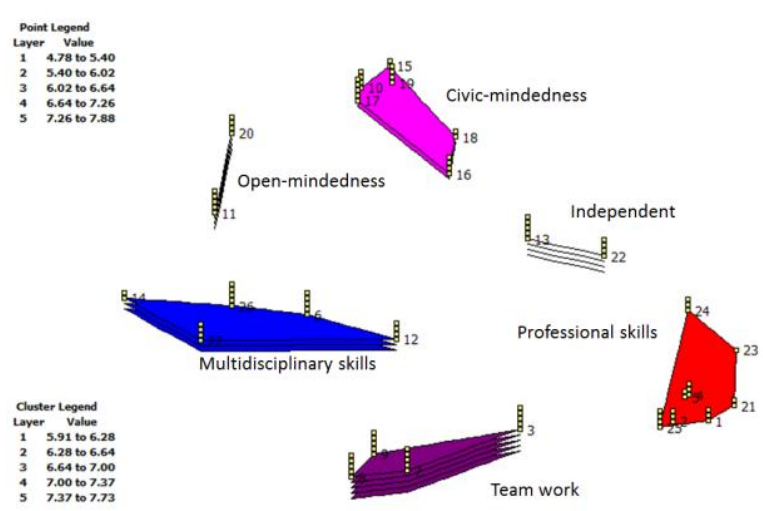

Fig. 4. Cluster rating map (performance).
Fig. 3 and Fig. 4 also show the various cut-off ratings for each of the levels of importance and performance. The use of such cluster rating maps is to provide a graphical representation of the clusters that at the same time includes useful information for easy understanding. To further understand the relationships between the perceived importance and students' performance, a pattern matching was accomplished.

In Fig. 5, the process of pattern matching was conducted to compare the importance and performance ratings. Pattern Matching utilizes a ladder graph format to visually display the degree of agreement between the ratings of the groups being compared. If the two groups are in complete agreement, the graph would consist of a series of parallel lines and closely resemble a ladder. To the extent that the two groups are not in agreement, the lines cross and represent varying amounts of agreement/disagreement. The amount of correlation (Pearson r) between the two groups is also reported to be .9 , which is considered to be highly correlated.

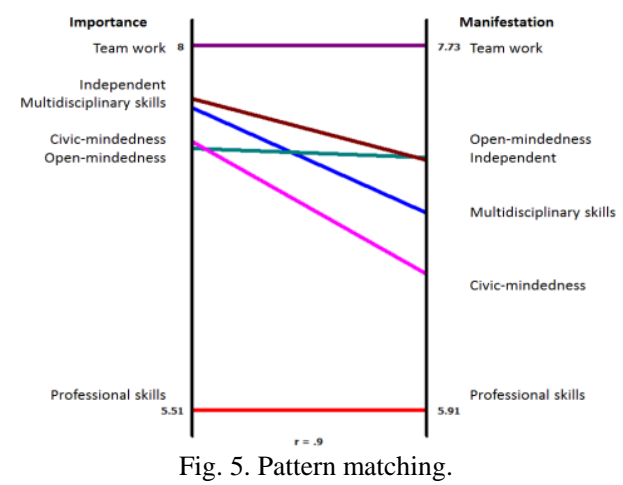

Lastly, similar to the results of Table II, Fig. 5 shows the variations between the perceived importance and the actual performance of the students. Such graphical representations easily portray the comparison between the importance and actual performance is an easy and quick to understood way.

\section{COnClusions}

Results of this study shows that the residential college outcome competencies seem to encompass a more varied perspective as compared to the outcome gains such as: general education, practical competence, and personal social gains of the student engagement model (Kuh, 2009). It is suggested that the current model of residential college provides the function of filling up the gap within the traditional educational system. This study shall look forward to further providing much needed empirical data for strengthening the concepts of residential colleges systems of learning. Furthermore, with the residential college learning model deemed as informal learning, which the outcome of such is hard to assess (difficult to apply on the existing college evaluation system). The development of a model of learning in residential colleges is suggested in order to make available a psychometrically sound tool for accessing how Taiwan college students learn in the current millennium.

\section{ACKNOWLEDGMENT}

This work was supported in part by the Taiwan Ministry of 
Science and Technology under Grant NSC 101-2410-H-004-168-MY2.

\section{REFERENCES}

[1] M. B. Ryan, "Residential colleges: An historical context," in Gateways: Residential Colleges and the Freshman Year Experience. Monograph Series Number 14, T. B. Smith Ed., Carolina: University of South Carolina, 1993, pp. 11-19.

[2] M. Durrani and K. Sohaib, "Bringing the residential college model to LUMS," 2009

[3] V. J. Caracelli, "Structured conceptualization: A framework for interpreting evaluation results," Evaluation and Program Planning, vol. 12, pp. 45-52, 2002.

[4] W. M. K. Trochim, "An introduction to concept mapping for planning and evaluation," A Special Issue of Evaluation and Program Planning, vol. 12, pp. 1-16, 1989

[5] W. M. K. Trochim and M. Kane, "Concept mapping: An introduction to structured conceptualization in health care," International Journal for Quality in Health Care, vol. 17, pp. 187-191, 2005.

[6] M. Kane and W. M. K. Trochim, Concept Mapping for Planning and Evaluation, CA: Sage Publications, 2007.

[7] K. M. Jackson and W. M. K. Trochim, "Concept mapping as an alternative approach for the analysis of open-ended survey responses," Organizational Research Methods, vol. 5, pp. 307-336, October 1, 2002.

[8] H. A. Leonard and C. Ashley, "Exploring the underlying dimensions of violence in print advertisements," Journal of Advertising, vol. 41, pp. 77-90, 2012.

[9] J. G. van Manen, J. H. Kamphuis, A. Goossensen, R. Timman, J. J. V. Busschbach, and R. Verheul, "In search of patient characteristics that may guide empirically based treatment selection for personality disorder patients: Concept map approach," Journal of Personality Disorders, vol. 26, pp. 481-497, 2012.

[10] W. M. K. Trochim and R. Linton, "Conceptualization for planning and evaluation," Evaluation and Program Planning, vol. 9, pp. 289-308, 1986.

[11] C. J. Drew, M. L. Hardman, and J. L. Hosp, Designing and Conducting Research in Education, Thousand Oaks, CA: Sage Publications, Inc., 2008.

[12] S. C. Weller and A. K. Romney, Systematic Data Collection, Newbury Park, CA: Sage Publications, 1988.

[13] A. P. M. Coxon, "Sorting data: Collection and analysis," University papers series on quantitative applications in the social sciences no. 07-127, Thousand Oaks, CA: Sage Publications, 1999.

[14] M. L. Davison, Multidimensional Scaling, New York: John Wiley \& Sons, Inc., 1983.
[15] J. B. Kruskal and M. Wish, Multidimensional Scaling, Beverly Hills, CA: Sage Publications, 1978

[16] M. R. Anderberg, Cluster Analysis for Applications, New York: Academic Press, Inc., 1973.

[17] B. Everitt, Cluster Analysis, 2nd ed., New York: Halsted Press, 1980.

[18] W. M. K. Trochim, The Concept System, Ithaca, NY: Concept Systems, 1989.

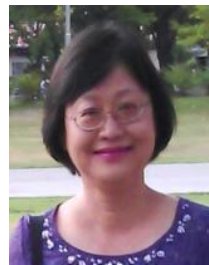

Yueh-Luen $\mathrm{Hu}$ is a $\mathrm{PhD}$ graduate of educational psychology from Indiana University, Bloomington, USA. Currently, she is a professor in the Department of Education, College of Education of the National Chengchi University, Taiwan.

She has been a member of the Examiners Committee of the Examination Yuan of ROC (Ministry of Examination), an examiner for the Examination Yuan of ROC (Ministry of Examination), structure interview method resource speaker for the Examination Yuan of ROC (Ministry of Examination), and a guest professor of the Department of Education, College of Education Summer School of Tohoku University, Japan.

Prof. Dr. Yueh-Luen Hu's current research interests include teachers selection interview, validity, meditation, and fuzzy models with applications in education, psychology, and measurement.

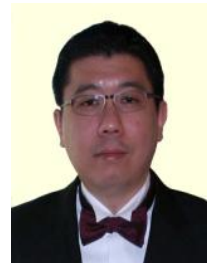

Gregory S. Ching received his $\mathrm{PhD}$ in education from the National ChengChi University, Taiwan, and he is currently an assistant professor at the Graduate School of Educational Leadership and Development in Fu Jen Catholic University, Taiwan.

Dr. Gregory S. Ching's research interests include technology and education, globalization, higher education issues, international student mobility, and student engagement.

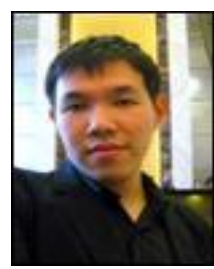

Chao-Hsiang Hung is a $\mathrm{PhD}$ candidate of the Department of Education, College of Education of the National ChengChi University, Taiwan. Currently, he is an instructor in the Department of Social Welfare and Social Work of the HsuanChuang University, Taiwan. $\mathrm{He}$ has been an analyst of the New Asia Institute of Human Metric, and a research assistant of the $\alpha \beta \gamma$ metric lab.

Mr. Chao-Hsiang Hun's current research interests include positive psychology, creativity, reading literacy, and advanced metric models with applications in education, psychology, and measurement. 